Pacific

Journal of

Mathematics

A CLASSIFICATION OF SPHERICAL CONJUGACY CLASSES IN GOOD CHARACTERISTIC

Giovanna CARnOvale 


\title{
A CLASSIFICATION OF SPHERICAL CONJUGACY CLASSES IN GOOD CHARACTERISTIC
}

\author{
Giovanna CARnOVAlE
}

\begin{abstract}
We classify spherical conjugacy classes in a simple algebraic group over an algebraically closed field of good, odd characteristic.
\end{abstract}

\section{Introduction}

When studying a transitive action of a group $G$, it is particularly interesting to understand when a given subgroup $B$ of $G$ acts with finitely many orbits. An important case of such a situation in the theory of algebraic groups is when $B$ is a Borel subgroup of a connected reductive algebraic group $G$. The $G$-spaces for which $B$ acts with finitely many orbits in this case are the so-called spherical homogeneous spaces, and they include important examples such as the flag variety $G / B$ and symmetric varieties. They are precisely those $G$-spaces for which the $B$ action has a dense orbit in the Zariski topology [Brion 1986; Grosshans 1992; Knop 1995; Vinberg 1986]. One may want to understand when homogeneous spaces that are relevant in algebraic Lie theory, such as nilpotent orbits in $\operatorname{Lie}(G)$ and conjugacy classes in $G$ for $G$ reductive, are spherical. Spherical nilpotent orbits in simple Lie algebras were classified in [Panyushev 1994; 1999] when the base field is $\mathbb{C}$ and in [Fowler and Röhrle 2008] when it is an algebraically closed field of good characteristic: They are precisely the orbits of type $r A_{1}$ for $r \geq 0$ in the simplylaced case and of type $r A_{1}+s \tilde{A}_{1}$ for $r, s \geq 0$ in the multiply-laced case. As for conjugacy classes, it is natural to use the interplay with the Bruhat decomposition, since this has proved to be a fruitful tool in the past. For instance, it is essential in describing regular conjugacy classes [Steinberg 1965], whose intersection with Bruhat cells is the subject of ongoing research [Ellers and Gordeev 2004; 2007]. This approach has led to two characterizations of the spherical conjugacy classes in a connected, reductive algebraic group $G$ over an algebraically closed field of zero or good, odd characteristic [Cantarini et al. 2005; Carnovale 2008; 2009]. The first one is given through a formula relating the dimension of a class $\mathbb{O}$ and the Weyl group element whose associated Bruhat cell intersects 0 in a dense subset.

MSC2000: primary 20E45, 20F55, 20G99; secondary 14M15.

Keywords: algebraic group, conjugacy class, Bruhat decomposition, spherical homogeneous space. 
The second one states that spherical conjugacy classes are exactly those classes intersecting only Bruhat cells corresponding to involutions in the Weyl group of $G$. These characterizations can be used to give a complete list of the spherical classes in $G$. This problem can be easily reduced to the case in which $G$ is simple, so we shall make this assumption from now on. The spherical conjugacy classes in a simple algebraic group over $\mathbb{C}$ have been classified in [Cantarini et al. 2005], making use of the classification of spherical nilpotent orbits. Spherical classes in type $G_{2}$ in good characteristic have been classified in [Carnovale 2009].

In the present paper, we complete the picture by classifying spherical classes in a simple algebraic group $G$ over a field of good, odd characteristic. In contrast to [Cantarini et al. 2005], this work is independent of the classification of spherical nilpotent orbits existing in the literature. Since Springer isomorphisms exist in good characteristic, it provides an elementary classification of spherical nilpotent orbits alternative to [Fowler and Röhrle 2008], where Kempf-Russeau theory is involved and where a computer program is needed to help deal with the exceptional types. The crucial tools in our method are just those conditions in the characterizations in [Cantarini et al. 2005; Carnovale 2008; 2009], whose proofs are general and rather short. The arguments used for this classification can also be transferred to the characteristic zero situation, providing an alternative, elementary approach to [Panyushev 1994; 1999], although by case-by-case considerations.

After fixing notation and recalling basic notions in Section 1, we introduce spherical conjugacy classes and their characterizations in Section 2. Section 3 provides the list of spherical conjugacy classes through a case-by-case analysis.

The result is as when the base field is $\mathbb{C}$ : In the simply-laced case, spherical conjugacy classes are, up to a central element, either semisimple or unipotent, and if $G$ is simply-connected, the centralizers of the semisimple ones are all subgroups of fixed points for an involution on $G$. By abuse of notation, we say that such classes are symmetric.

In type $G_{2}$, spherical conjugacy classes are again either semisimple or unipotent but, as in types $B_{n}$ and $C_{n}$, there are spherical semisimple classes that are not symmetric. Just as in other situations involving spherical homogeneous spaces (for example, in the description of maximal spherical ideals of Borel subalgebras [Panyushev and Röhrle 2005]), the doubly-laced case is slightly more involved. The new phenomenon in the present situation is that there appear spherical classes that are neither semisimple nor unipotent.

\section{Notation}

Let $G$ be a connected reductive algebraic group over an algebraically closed field $k$ of good odd characteristic [Springer and Steinberg 1970, Section I.4]. In Section 3, 
we will restrict to the case of simple $G$. When we consider an integer as an element in $k$, we mean its image in the prime field of $k$. We denote by $\Phi$ and $\Phi^{+}$the root system and the set of positive roots relative to a fixed Borel subgroup $B$ and a maximal torus $T$ of $G$; denote by $\Delta=\left\{\alpha_{1}, \ldots, \alpha_{n}\right\}$ the corresponding set of simple roots. We number the simple roots as in [Bourbaki 1981, planches I-IX]. Denote the highest positive root by $\beta_{1}$. For a root $\alpha$, we denote the elements of the associated root subgroup $X_{\alpha}$ by $x_{\alpha}(t)$, and we put $X_{\alpha}^{\prime}=X_{\alpha} \backslash\{1\}$. We denote the maximal unipotent subgroup of $B$ by $U$.

For elements in $T$ in exceptional simple groups, we use the notation in [Steinberg 1968, Lemma 19], that is, every element in $T$ can be expressed as a product of $h_{\alpha_{i}}\left(t_{i}\right)$ for $i=1, \ldots, n$ and nonzero $t_{i} \in k$, with uniqueness if the group is simply connected. The $h_{\alpha_{i}}\left(t_{i}\right)$ satisfy the commutation relations

$$
h_{\alpha_{i}}(t) x_{\beta}(r) h_{\alpha_{i}}\left(t^{-1}\right)=x_{\beta}\left(t^{\left\langle\beta, \alpha_{i}\right\rangle} r\right) \quad \text { for } \beta \in \Phi \text { and } t, r \in k,
$$

where $\langle\beta, \alpha\rangle=\beta\left(h_{\alpha}\right)$ as usual; see [Steinberg 1968].

When $G$ is simple of type $A_{n}, B_{n}, C_{n}$ or $D_{n}$, we work with the corresponding matrix groups, and we choose $G$ and $T$ so that the elements in $T$ are diagonal. Let $X_{1}, \ldots, X_{l}$ be square matrices of size $n_{j} \geq 1$ for $j=1, \ldots, l$. By $\operatorname{diag}\left(X_{1}, \ldots, X_{l}\right)$ we mean the square matrix of size $\sum_{j} n_{j}$ with the blocks $X_{1}, \ldots, X_{l}$ along its diagonal. As usual, $E_{i j}$ is a square matrix with the entry 1 in the $i$-th row and $j$-th column and all other entries 0 . We denote by ${ }^{t} M$ the transpose of a matrix $M$.

We put $W=N(T) / T$, and $s_{\alpha}$ indicates the reflection corresponding to the root $\alpha$. Given an element $w \in W$, we denote by $\dot{w}$ a representative of $w$ in $N(T)$.

Let $\ell$ denote the usual length function on $W$, and let $\operatorname{rk}(1-w)$ denote the rank of the endomorphism $1-w$ in the geometric representation of $W$.

We shall frequently use these properties of the Bruhat decomposition of $G$ (see [Bourbaki 1981, IV.2.4]):

$$
\begin{aligned}
X_{-\alpha}^{\prime} & \subset X_{\alpha}^{\prime} s_{\alpha} T X_{\alpha}^{\prime} \subset B s_{\alpha} B \quad \text { for all } \alpha \in \Phi^{+}, \\
B w B w^{\prime} B & =B w w^{\prime} B \quad \text { if } \ell\left(w w^{\prime}\right)=\ell(w)+\ell\left(w^{\prime}\right) .
\end{aligned}
$$

Given an element $x \in G$, we denote by $O_{x}$ the conjugacy class of $x$ in $G$ and by $H_{x}$ the centralizer of $x$ in $H \leq G$. Denote by $Z(K)$ the center of an algebraic group $K$ and by $K^{\circ}$ its identity component.

For the dimension of unipotent conjugacy classes in arbitrary good characteristic, see [Carter 1985, Chapter 13] and [Premet 2003, Theorem 2.6].

For a conjugacy class $O$ in $G$, we denote by $\mathscr{V}$ the set of its $B$-orbits.

\section{Characterizations through the Bruhat decomposition}

Here we introduce our characterizations of spherical conjugacy classes. 
Definition 2.1. Let $G$ be a connected reductive algebraic group. A homogeneous $G$-space $X$ is spherical if it has a dense orbit for a Borel subgroup of $G$.

It is well known [Brion 1986; Grosshans 1992; Knop 1995; Vinberg 1986] that $O$ is a spherical conjugacy class in $G$ if and only if its set of $B$-orbits $\mathscr{V}$ is finite.

Since $G=\bigcup_{w \in W} B w B$, for every class $\mathcal{O}$ there is a natural map $\phi: \mathscr{V} \rightarrow W$ associating to $v \in \mathscr{V}$ the element $w$ in the Weyl group of $G$ for which $v \subset B w B$. Besides, there is a unique $w \in W$ for which $B w B \cap \mathcal{O}$ is dense in $\mathbb{O}$, and this element, which we denote by $w_{\mathbb{O}}$, is maximal in $\operatorname{Im}(\phi)$ with respect to the Bruhat ordering [Cantarini et al. 2005, page 32].

There are two characterizations of spherical classes in $G$.

Theorem 2.2 [Cantarini et al. 2005, Theorem 25; Carnovale 2008, Theorem 4.4]. A class 0 in a connected reductive algebraic group $G$ over an algebraically closed field of zero or good odd characteristic is spherical if and only if there exists $v$ in $\mathscr{V}$ such that $\ell(\phi(v))+\operatorname{rk}(1-\phi(v))=\operatorname{dim} \mathbf{O}$. If this is the case, $v$ is the dense B-orbit and $\phi(v)=w_{0}$.

Theorem 2.3 [Carnovale 2008, Theorem 2.7; Carnovale 2009, Theorem 5.7]. A class 0 in a connected reductive algebraic group $G$ over an algebraically closed field of zero or odd, good characteristic is spherical if and only if $\operatorname{Im}(\phi)$ contains only involutions in $W$.

Since all Borel subgroups and all maximal tori are $G$-conjugate, the statement in Theorem 2.3 is independent of the choice of $B$ and $T$. By abuse of notation, we say that $g \in G$ is spherical if its class $\mathrm{O}_{g}$ is.

Remark 2.4. Let $g \in G$. The $B$-orbits in $O_{g}$ are in one-to-one correspondence with the $\left(B, G_{g}\right)$-double cosets in $G$. Therefore if $x \in G$ is such that $G_{x}=G_{g}$, then $\mathscr{O}_{g}$ is spherical if and only if $\mathscr{O}_{x}$ is. In particular, if $g^{2} \in Z(G)$, then $g$ and $x$ are semisimple. If $G$ is affine, by [Borel 1969, Proposition 9.1] the orbit map is separable, so the symmetric variety $G / G_{g}=G / G_{x}$ is $G$-equivariantly isomorphic to $O_{g}$ and $O_{x}$. By [Springer 1985, Corollary 4.3], the class $O_{x}$ is spherical. Motivated by this, we abuse notation when $G_{x}=G_{g}$ and $g^{2} \in Z(G)$ by saying that $O_{x}$ is a symmetric conjugacy class.

Remark 2.5. Regular classes in a reductive algebraic group whose semisimple quotient is not of type $r A_{1}$ cannot be spherical. By [Steinberg 1965, Theorem 8.1], regular classes intersect Bruhat cells corresponding to Coxeter elements.

We will frequently use the following observation.

Lemma 2.6. Let $G$ be a connected reductive algebraic group, let $T$ be a maximal torus in $G$, and let $H$ be a closed connected reductive subgroup of $G$ containing $T$. Let $x \in H$ and suppose that $O_{x}$ is spherical. Then the H-conjugacy class of $x$ is spherical. 
Proof. Let $B_{H}$ be a Borel subgroup of $H$ containing $T$, and let $B$ be a Borel subgroup of $G$ containing $B_{H}$. Let $y$ lie in the $H$-conjugacy class of $x$. For some $\dot{w} \in N_{H}(T)=N(T) \cap H$ and for some $b_{1}, b_{2} \in B_{H} \leq B$, we have

$$
y=b_{1} \dot{w} b_{2} \in B_{H} N_{H}(T) B_{H} \subset B N(T) B .
$$

Since $y \in \mathcal{O}_{x}$, we have $\dot{w}^{2} \in T$ by Theorem 2.3. As this holds for every $y \in H$, the $H$-class of $x$ satisfies the sufficient condition provided by Theorem 2.3.

As a first application of Lemma 2.6 we have the following statement.

Lemma 2.7. Let $G$ be a connected reductive algebraic group. Let $g \in G$ with Jordan decomposition $g=s u$. If $\mathrm{O}_{g}$ is spherical, then $\mathrm{O}_{s}$ and $\mathrm{O}_{u}$ are spherical in $G$ and the $G_{s}^{\circ}$-class of $u$ is spherical.

Proof. It is well known that $G_{g}=G_{s} \cap G_{u}$. Therefore, if for a Borel subgroup $B$ of $G$ there are finitely many $\left(B, G_{g}\right)$ double cosets in $G$, there are finitely many $\left(B, G_{s}\right)$ double cosets and $\left(B, G_{u}\right)$ double cosets in $G$. Thus if $O_{g}$ is spherical, then $\mathrm{O}_{s}$ and $\mathrm{O}_{u}$ are also spherical. For the last statement, by [Humphreys 1995, Section 1.12], we have $u \in G_{s}^{\circ}$, and we may apply Lemma 2.6 with $H=G_{s}^{\circ}$.

The next lemma helps show that certain classes in a group are not spherical.

Lemma 2.8. Let $G$ be a connected reductive algebraic group, let $T$ be a maximal torus in $G$, and let $H$ be a closed, connected, reductive subgroup of $G$ containing $T$ such that its semisimple part is not of type $r A_{1}$. Let $x \in H$ and suppose that the $H$-conjugacy class of $x$ is regular. Then $O_{x}$ is not spherical.

Proof. This is obtained by combining Lemma 2.6 with Remark 2.5 .

\section{The classification}

From now on $G$, will be a simple algebraic group. We aim at a classification of spherical conjugacy classes in $G$ in good odd characteristic. The main tools in our classification will be the sufficient condition in Theorem 2.2 and the necessary condition in Theorem 2.3.

If $\pi: G_{1} \rightarrow G_{2}$ is a central isogeny between two simple algebraic groups, a conjugacy class $O_{g}$ in $G_{1}$ is spherical if and only if $\pi\left(O_{g}\right)$ is spherical. Indeed, let $x \in G_{1}$, with $G_{1, x}$ its centralizer in $G_{1}$ and $G_{2, x}$ the centralizer of $\pi(x)$ in $G_{2}$. Also suppose $B_{1}$ is a Borel subgroup of $G_{1}$. Then $\pi\left(B_{1}\right)$ is a Borel subgroup of $G_{2}$, and the $\left(B_{1}, G_{1, x}\right)$-double cosets of $G_{1}$ are in one-to-one correspondence with the $\left(B_{2}, G_{2, x}\right)$-double cosets of $G_{2}$. For this reason it is enough to provide the classification for one representative for each isogeny class of simple groups.

By Remark 2.4, if $x, y \in G$ and $x y^{-1}$ is central, then $O_{x}$ is spherical if and only if $O_{y}$ is. Thus it is enough to provide the classification up to a central element. 
If $G$ is of type $G_{2}$, Carnovale [2009, Section 2.1] gives the classification in good characteristic; we provide it here for completeness.

\section{Type $G_{2}$.}

Theorem 3.1. Let $G$ be of type $G_{2}$. The spherical classes are either semisimple or unipotent. The semisimple ones are represented by $h_{\alpha_{1}}(-1)$ and $h_{\alpha_{1}}(\zeta)$ for $\zeta$ a fixed primitive third root of 1 . The unipotent ones are those of type $A_{1}$ and $\tilde{A}_{1}$.

Type $\boldsymbol{A}_{\boldsymbol{n}}$. In this section $G=\mathrm{SL}_{n+1}(k), B$ is the subgroup of upper triangular matrices, $T$ is the subgroup of diagonal matrices in $G$, and $U$ is the unipotent radical of $B$. For a positive root $\alpha=\alpha_{i}+\alpha_{i+1}+\cdots+\alpha_{j}$ we have

$$
X_{\alpha}=\left\{1+t E_{i, j+1}, t \in k\right\} \quad \text { and } \quad X_{-\alpha}={ }^{t} X_{\alpha} \quad \text { for every } \alpha \in \Phi .
$$

Theorem 3.2. If $n=1$, all classes in $G$ are spherical. If $n \geq 2$, the spherical classes in $G$ are either semisimple or unipotent up to a central element. The semisimple ones are those corresponding to matrices with at most two distinct eigenvalues, and they are all symmetric. The unipotent ones are those associated with the partitions $\left(2^{m}, 1^{n+1-2 m}\right)$ for $m=1, \ldots,[(n+1) / 2]$.

Proof. If $n=1$, all Bruhat cells correspond to involutions in $W$, so every class is spherical by Theorem 2.3.

Unipotent classes. Let $n \geq 2$, and let $\mathrm{O}=\mathrm{O}_{u}$ be a unipotent class. By Jordan theory, we may assume that $u=x_{\alpha_{1}}\left(c_{1}\right) \cdots x_{\alpha_{n}}\left(c_{n}\right)$ with $c_{i} \in\{0,1\}$. Then $u$ lies in the connected reductive subgroup $H$ generated by $T$ and by $X_{ \pm \alpha_{i}}$ for all $i$ such that $c_{i}=1$. By [Steinberg 1965, Lemma 3.2 and Theorem 3.3], $u$ is regular in $H$. Lemma 2.8 implies that if $\mathrm{O}_{u}$ is spherical then $c_{i} c_{i+1}=0$, so its associated partition is of type $\left(2^{m}, 1^{n+1-2 m}\right)$. Conversely, let $O_{j}$ be the unipotent class corresponding to $\left(2^{j}, 1^{n+1-2 j}\right)$, with $2 j \leq n+1$. Let $\beta_{i}=\alpha_{i}+\cdots+\alpha_{n-i+1}$ for $i=1, \ldots, j$. The element $x_{-\beta_{1}}(1) \cdots x_{-\beta_{j}}$ (1) lies in $\mathfrak{O}_{j}$. By (1) and (2) this element lies in $B s_{\beta_{1}} \cdots s_{\beta_{j}} B$, so its $B$-orbit satisfies the condition in Theorem 2.2, and thus $\mathrm{O}_{j}$ is spherical.

Semisimple classes. Let $s=\operatorname{diag}\left(\lambda_{1} I_{n_{1}}, \lambda_{2} I_{n_{2}}, \ldots, \lambda_{l} I_{n_{l}}\right)$ for distinct scalars $\lambda_{i}$. If $l>2$, then $s$ is conjugate to $t=\operatorname{diag}\left(\lambda_{1}, \lambda_{2}, \lambda_{3}, t_{1}\right)$ for some invertible diagonal submatrix $t_{1}$. Then $t$ lies in the connected reductive subgroup $H=\left\langle T, X_{ \pm \alpha_{1}}, X_{ \pm \alpha_{2}}\right\rangle$, and it is regular therein. It follows from Lemma 2.8 that if $O_{s}$ is spherical semisimple, then $s$ has at most 2 eigenvalues. Conversely, suppose that $s \in T$ has 2 eigenvalues. We may assume $s=\operatorname{diag}\left(\lambda I_{m}, \mu I_{n+1-m}\right)$. Let $\zeta$ be a primitive $2(n+1)$-st root of unity if $n+1-m$ is odd, and let $\zeta=1$ if $n+1-m$ is even. Let also $s_{0}=\operatorname{diag}\left(\zeta I_{m},-\zeta I_{n+1-m}\right)$. Then $s_{0}^{2} \in Z(G)$ and $G_{s}=G_{s_{0}}$. By Remark 2.4 the class $O_{s}$ is symmetric and hence spherical. 
Mixed classes. We now show that there is no spherical element $x$ with Jordan decomposition $x=s u$ such that $s \notin Z(G)$ and $u \neq 1$. Were this the case, we could assume by Lemma 2.7 that $s=\operatorname{diag}\left(\lambda I_{m}, \mu I_{n+1-m}\right)$ with $m \geq 2$ and that $u \in U \cap G_{s}=\left\langle X_{\alpha_{i}}, i \neq m\right\rangle$.

We could then choose $u=x_{\alpha_{1}}\left(t_{1}\right) \cdots x_{\alpha_{m-1}}\left(t_{m-1}\right) x_{\alpha_{m+1}}\left(t_{m+1}\right) \cdots x_{\alpha_{n}}\left(t_{n}\right)$ with $t_{i} t_{i+1}=0$ because $u$ is spherical by Lemma 2.7. If $u$ is nontrivial, we may assume that $t_{m-1}$ or $t_{m+1}$ is nonzero. Put $J=\left\{i \mid t_{i} \neq 0\right\}$ and $H=\left\langle T, X_{ \pm \alpha_{m}}, X_{ \pm \alpha_{i}}\right\rangle_{i \in J}$. Then $s u$ is regular in $H$. Since $H$ contains at least a subgroup of type $A_{2}$ we may conclude using Lemma 2.8.

Type $\boldsymbol{C}_{\boldsymbol{n}}$. Let us view $G=\mathrm{Sp}_{2 n}(k)$ as the subgroup of $\mathrm{GL}_{2 n}(k)$ of matrices preserving the bilinear form whose matrix is $\left(\begin{array}{rr}0 & I \\ -I & 0\end{array}\right)$ in the canonical basis of $k^{2 n}$. We choose $B$ as the subgroup of $G$ of matrices of the form $\left(\begin{array}{ccc}A & A X \\ 0 & { }^{t} A^{-1}\end{array}\right)$, where $A$ is an invertible upper triangular matrix, ${ }^{t} A^{-1}$ is its inverse transpose, and $X$ is a symmetric matrix. The torus $T$ is the subgroup of diagonal matrices in $B$. We have

$$
\begin{aligned}
& X_{\alpha_{i}}=\left\{I+t E_{i, i+1}-t E_{n+i+1, n+i}, t \in k\right\} \quad \text { for } i=1, \ldots, n-1, \\
& X_{\alpha_{n}}=\left\{I+t E_{n, 2 n}, t \in k\right\},
\end{aligned}
$$

and $X_{-\alpha}={ }^{t} X_{\alpha}$ for every $\alpha \in \Phi$. We recall that if $g, h \in \mathrm{Sp}_{2 n}(k)$ are $\mathrm{GL}_{2 n}(k)$ conjugate they are $\mathrm{Sp}_{2 n}(k)$-conjugate [Springer and Steinberg 1970, IV.2.15(ii)]. It is well known that unipotent classes in $G$ are parametrized through Jordan theory by partitions where odd terms occur pairwise [Humphreys 1995, Section 7.11].

Theorem 3.3. Let $G=\operatorname{Sp}_{2 n}(k)$ for $n \geq 2$. The nontrivial spherical semisimple classes are represented by $\sigma_{l}=\operatorname{diag}\left(-I_{l}, I_{n-l},-I_{l}, I_{n-l}\right)$ for $l=1, \ldots, n-1$; by $a_{\lambda}=\operatorname{diag}\left(\lambda I_{n}, \lambda^{-1} I_{n}\right)$; and, up to a sign, by $c_{\lambda}=\operatorname{diag}\left(\lambda, I_{n-1}, \lambda^{-1}, I_{n-1}\right)$ for $\lambda \in k$ with $\lambda^{2} \neq 0,1$. The unipotent ones are those associated with the partitions $\left(2^{m}, 1^{2 n-2 m}\right)$ for $m=1, \ldots, n$. The spherical classes that are neither semisimple nor unipotent up to a sign are represented by the elements $\sigma_{l} u$, where $u \in G_{\sigma_{l}} \cong$ $\mathrm{Sp}_{2 l}(k) \times \mathrm{Sp}_{2 n-2 l}(k)$ is unipotent and corresponds to the partition $\left(2,1^{2 n-2}\right)$.

Proof. Semisimple classes. Let $s \in T$, and let $\Lambda$ be the set of eigenvalues of $s$.

Let us first suppose that $|\Lambda| \geq 4$. If $n=2$, then $s$ is a regular element, and hence it is not spherical. Let $n \geq 3$.

If $\{ \pm 1\} \subset \Lambda$, then $s$ is conjugate to $s^{\prime}=\operatorname{diag}\left(\lambda, 1,-1, t, \lambda^{-1}, 1,-1, t^{-1}\right)$ for some invertible diagonal submatrix $t$ and some nonzero $\lambda \in k$ with $\lambda^{2} \neq 1$.

If $|\{ \pm 1\} \cap \Lambda|=1$, then, since eigenvalues come with their inverse, $|\Lambda| \geq 5$ and $s$ is conjugate to $s^{\prime}=\operatorname{diag}\left(\lambda, \mu, \pm 1, t, \lambda^{-1}, \mu^{-1}, \pm 1, t^{-1}\right)$ for some invertible diagonal submatrix $t$ and some $\lambda \neq \mu \in k$ with $\lambda^{2} \neq 1 \neq \mu^{2}$.

If $\{ \pm 1\} \cap \Lambda=\varnothing$, then either $|\Lambda| \geq 6$ or there are two reciprocally inverse eigenvalues with multiplicity at least 2 . In both cases, the matrix $s$ is conjugate to 
$s^{\prime}=\operatorname{diag}\left(\lambda, \mu, v, t, \lambda^{-1}, \mu^{-1}, v^{-1}, t^{-1}\right)$ for some invertible diagonal submatrix $t$ and some distinct $\lambda, \mu, v \in k$ with $\lambda^{2}, \mu^{2}, v^{2} \neq 1$ and $v$ possibly equal to $\lambda^{-1}$.

In all these cases, the element $s^{\prime}$ is regular in $H=\left\langle T, X_{ \pm \alpha_{1}}, X_{ \pm \alpha_{2}}\right\rangle$; therefore by Lemma 2.8 the class $O_{s}$ cannot be spherical.

Let us now suppose that $|\Lambda|=3$. Then $\Lambda=\left\{\eta, \lambda, \lambda^{-1}\right\}$ with $\eta^{2}=1$ and $\lambda^{2} \neq 1$. If the multiplicity of $\lambda^{ \pm 1}$ is greater than 1 , then $s$ is conjugate to some $r^{\prime}=$ $\operatorname{diag}\left(\lambda, \lambda^{-1}, 1, r_{1}, \lambda^{-1}, \lambda, 1, r_{1}^{-1}\right)$ with $r_{1}$ an invertible, diagonal submatrix. The element $r^{\prime}$ lies and is regular in the subgroup $H$ above described. By Lemma 2.8 the class $O_{s}$ cannot be spherical. On the other hand, if $\Lambda=\left\{\lambda^{ \pm 1}, 1\right\}$ with the multiplicity of $\lambda^{ \pm 1}$ equal to 1 , then $\mathscr{O}_{s}$ is spherical. Indeed, the representative of such a class in [Cantarini et al. 2005, Theorem 15, page 42] works also in odd characteristic and its $B$-orbit satisfies the condition of Theorem 2.2.

Now assume that $|\Lambda|=2$. Then either $\Lambda=\{ \pm 1\}$ so that $O_{s}$ is symmetric, or $\Lambda=\left\{\lambda, \lambda^{-1}\right\}$ for $\lambda^{2} \neq 1$ so that $s$ is conjugate to $a_{\lambda}=\operatorname{diag}\left(\lambda I_{n}, \lambda^{-1} I_{n}\right)$, whose centralizer is independent of $\lambda$ in the given range. Since $a_{\zeta}^{2} \in Z(G)$ if $\lambda=\zeta$ is a primitive fourth root of 1 , we may apply Remark 2.4 and conclude that $a_{\lambda}$ is spherical.

Unipotent classes. Let $\mathcal{O}_{u}$ be a unipotent class and let $\underline{\lambda}$ be its associated partition. Let $\underline{\mu}=\left(\mu_{1}, \ldots, \mu_{l}\right)$ be obtained by taking a representative of each term occurring pairwise in $\underline{\lambda}$ and let $\underline{v}=\left(v_{1}, \ldots, v_{m}\right)$ be obtained by taking the remaining even terms without repetition in $\underline{\lambda}$, so that $2 n=|\underline{v}|+2|\underline{\mu}|$. A representative $u^{\prime}$ of $\mathrm{O}_{u}$ can be taken in the subgroup isomorphic to

$$
\operatorname{Sp}_{2 \mu_{1}}(k) \times \cdots \times \operatorname{Sp}_{2 \mu_{l}}(k) \times \operatorname{Sp}_{v_{1}}(k) \times \cdots \times \operatorname{Sp}_{v_{m}}(k)
$$

obtained by repeating the immersion of $\mathrm{Sp}_{2 d_{1}}(k) \times \mathrm{Sp}_{2 d_{2}}(k)$ into $\mathrm{Sp}_{2\left(d_{1}+d_{2}\right)}(k)$ given by

$$
\left(\left(\begin{array}{ll}
A_{1} & B_{1} \\
C_{1} & D_{1}
\end{array}\right),\left(\begin{array}{ll}
A_{2} & B_{2} \\
C_{2} & D_{2}
\end{array}\right)\right) \mapsto\left(\begin{array}{llll}
A_{1} & & B_{1} & \\
& A_{2} & & B_{2} \\
C_{1} & & D_{1} & \\
& C_{2} & & D_{2}
\end{array}\right) .
$$

The component of $u^{\prime}$ in $\operatorname{Sp}_{v_{j}}(k)$ corresponds to the partition $\left(v_{j}\right)$ and is thus regular in $\operatorname{Sp}_{v_{j}}(k)$, whereas the component of $u^{\prime}$ in $\operatorname{Sp}_{2 \mu_{i}}(k)$ can be taken to lie and be regular in the subgroup isomorphic to $\mathrm{SL}_{\mu_{i}}(k)$ obtained by the immersion mapping $M$ to $\operatorname{diag}\left(M,{ }^{t} M^{-1}\right)$. Therefore, $u^{\prime}$ is regular in the semisimple group

$$
\mathrm{SL}_{\mu_{1}}(k) \times \cdots \times \mathrm{SL}_{\mu_{l}}(k) \times \operatorname{Sp}_{v_{1}}(k) \times \cdots \times \mathrm{Sp}_{v_{m}}(k) .
$$

By Remark 2.5 if $u$ is spherical, we have $\mu_{i} \leq 2$ and $v_{j} \leq 2$ for every $i$ and $j$. Conversely, let $\underline{\lambda}=\left(2^{j}, 1^{2 n-2 j}\right)$, and let $\mathcal{O}_{j}$ be the unipotent class associated with $\underline{\lambda}$. Let $\beta_{q}=2 \alpha_{q}+\cdots+2 \alpha_{n-1}+\alpha_{n}$ for $q=1, \ldots, n-1$ and $\beta_{n}=\alpha_{n}$. The 
element

$$
x_{-\beta_{1}}(1) \cdots x_{-\beta_{j}}(1)=\left(\begin{array}{ll}
I_{n} & \\
X_{j} & I_{n}
\end{array}\right) \quad \text { with } X_{j}=\operatorname{diag}\left(I_{j}, 0_{n-j}\right)
$$

lies in $B s_{\beta_{1}} \cdots s_{\beta_{j}} B$ by (1) and (2). Since it also lies in $\mathcal{O}_{j}$, its $B$-orbit satisfies the condition in Theorem 2.2 for $O_{j}$; see [Cantarini et al. 2005, Theorem 12, page 36]. Thus, $O_{j}$ is spherical.

Mixed classes. Let $g=s u$ be the Jordan decomposition of a spherical element in $G$ with $s \notin Z(G)$ and $u \neq 1$. Then $\mathscr{O}_{s}$ is spherical and we may assume $s$ equals $a_{\lambda}, c_{\lambda}$ or $\sigma_{l}$ for some $l$. The case $s=a_{\lambda}$ is ruled out because $\operatorname{dim} 0_{a_{\lambda} u}>\operatorname{dim} O_{a_{\lambda}}=\operatorname{dim} B$, so $\mathrm{O}_{a_{\lambda} u}$ cannot have a dense $B$-orbit.

Assume that $s=c_{\lambda}$. Then $u \in G_{s} \cong k^{*} \times \operatorname{Sp}_{2 n-2}(k)$ and it is spherical therein, so it corresponds to a partition $\left(2^{m}, 1^{2 n-2-2 m}\right)$ for some $m \geq 1$. The class $\mathbb{O}_{c_{\lambda} u}$ is represented by $c_{\lambda} x_{\beta_{2}}(1) \cdots x_{\beta_{m+1}}(1)$, with notation as before. Such an element is regular in the subgroup $H=\left\langle T, X_{ \pm \alpha_{1}}, X_{ \pm \beta_{i}}, i=2, \ldots, m+1\right\rangle$. This case is thus excluded by Lemma 2.8 because the semisimple part of $H$ is of type $C_{2} \times(m-1) A_{1}$.

It follows that $s=\sigma_{l}$ for some $l$. Then $G_{s}$ is generated by $X_{ \pm \alpha_{i}}$ for $i \neq l$ and $X_{ \pm \beta_{l}}$. We have $u=\left(u_{1}, u_{2}\right) \in G_{s} \cong \mathrm{Sp}_{2 l}(k) \times \mathrm{Sp}_{2 n-2 l}(k)$, and it is spherical therein. Then $u_{1}$ and $u_{2}$ are spherical in the respective components. We claim that $u_{1}$ and $u_{2}$ cannot be both nontrivial. If on the contrary $u_{1}$ corresponded to the partition $\underline{\lambda}=\left(2^{a}, 1^{2 l-2 a}\right)$ and $u_{2}$ corresponded to the partition $\underline{\mu}=\left(2^{b}, 1^{2 n-2 l-2 b}\right)$ with $a, b \geq 1$, the $G_{s}$-class of $u_{1}$ would be represented by $u_{1}^{\prime}=\bar{x}_{\beta_{l-a+1}}(1) \cdots x_{\beta_{l}}(1)$ and the $G_{s}$-class of $u_{2}$ would be represented by $u_{2}^{\prime}=x_{\beta_{l+1}}(1) \cdots x_{\beta_{l+b}}(1)$. It is not hard to verify that $\sigma_{l} u_{1}^{\prime} u_{2}^{\prime}$ is regular in $\left\langle T, X_{ \pm \alpha_{l}}, X_{ \pm \beta_{i}}, i=l-a+1, \ldots, l+b\right\rangle$, whose semisimple part is of type $(a+b-2) A_{1}+C_{2}$. By Lemma 2.8 this option is excluded, and we have $a+b \leq 1$; hence at least one of the $u_{i}$ is trivial.

There is no loss of generality in assuming that $u_{1}=1$. We claim that the partition $\underline{\mu}=\left(2^{b}, 1^{2 n-2 l-2 b}\right)$ associated with $u_{2}$ has no repeated 2 . Let $b=2 h+j$ with $\bar{j}=0,1$ according to the parity of $b$, and assume that $h \geq 1$. The $G_{s}$-class of $u_{2}$ is represented by $u_{2}^{\prime}=x_{\alpha_{l+1}}(1) x_{\alpha_{l+3}}(1) \cdots x_{\alpha_{l+2 h-1}}(1) x_{\beta_{l+2 h+1}}(j)$. The element $\sigma u_{2}^{\prime}$ is regular in $\left\langle T, X_{ \pm \alpha_{l}}, X_{ \pm \alpha_{l+1}}, X_{ \pm \alpha_{l+3}}, \ldots, X_{ \pm \alpha_{2 h-1}}, X_{ \pm \beta_{2 h+1}}(j)\right\rangle$, whose semisimple part is of type $A_{2} \times h \tilde{A}_{1} \times j A_{1}$, where $\tilde{A_{1}}$ corresponds to a short root. By Lemma 2.8 the claim is proved.

Conversely, for all classes of type $\sigma_{l} u$ with $u \in G_{\sigma_{l}}$ corresponding to the partition $\left(2,1^{2 n-2}\right)$, the representative in [Cantarini et al. 2005, Theorem 21, page 50] is defined in odd characteristic and its $B$-orbit satisfies the condition of Theorem 2.2.

Type $\boldsymbol{D}_{\boldsymbol{n}}$. Let $n \geq 4$, and view $\mathrm{O}_{2 n}(k)$ as the subgroup of $\mathrm{GL}_{2 n}(k)$ of matrices preserving the bilinear form whose matrix is $\left(\begin{array}{ll}0 & I \\ I & 0\end{array}\right)$ in the canonical basis of $k^{2 n}$, 
so that $G=\mathrm{SO}_{2 n}(k)$ is viewed as the subgroup of such matrices of determinant 1 . We choose $B$ as the subgroup of $G$ of matrices of the form

$$
\left(\begin{array}{cc}
A & A X \\
0 & { }^{t} A^{-1}
\end{array}\right)
$$

where $A$ is an invertible upper triangular matrix, ${ }^{t} A^{-1}$ is its inverse transpose and $X$ is a skew-symmetric matrix. We fix $T \subset B$ as its subgroup of diagonal matrices.

We have

$$
\begin{aligned}
& X_{\alpha_{i}}=\left\{I+t E_{i, i+1}-t E_{n+i+1, n+i}, t \in k\right\} \quad \text { for } i=1, \ldots, n-1, \\
& X_{\alpha_{n}}=\left\{I+t E_{n-1,2 n}-t E_{n, 2 n-1}, t \in k\right\}
\end{aligned}
$$

and ${ }^{t} X_{-\alpha}=X_{\alpha}$ for every $\alpha \in \Phi$.

We recall that if $g, h \in G$ are $\mathrm{GL}_{2 n}(k)$-conjugate, they are $\mathrm{O}_{2 n}(k)$-conjugate [Springer and Steinberg 1970, IV.2.15(ii)] but not necessarily $G$-conjugate. However, conjugation by an element in $\mathrm{O}_{2 n}(k)$ determines an automorphism $\psi$ of $G$, so if $h=\psi(g)$, the class $\mathbb{O}_{g}$ is spherical if and only if $\psi\left(\mathbb{O}_{g}\right)=\mathbb{O}_{\psi(g)}=\mathbb{O}_{h}$ is. For this reason, in what follows we will sometimes replace an element $g \in G$ by a $\mathrm{GL}_{2 n}(k)$-conjugate $h$ lying in $G$.

To list a representative for each spherical conjugacy class, we will then have to verify whether an $\mathrm{O}_{2 n}(k)$-class splits into two $G$-classes or not. We recall that such a class splits into two classes if and only if the $\mathrm{O}_{2 n}(k)$-centralizer of a representative is contained in $G$.

It is well known that the even terms occur pairwise in the partition $\underline{\lambda}$ associated with a unipotent conjugacy class in $G$ via Jordan theory. Moreover, a unipotent $\mathrm{O}_{2 n}(k)$-class splits into two $G$-classes only if $n$ is even and the associated partition has only even terms [Humphreys 1995, Section 7.11].

Theorem 3.4. Let $G=\mathrm{SO}_{2 n}(k)$ for $n \geq 4$. The spherical classes in $G$ are either semisimple or unipotent up to a central element. The nontrivial semisimple ones are those represented by

$$
\begin{array}{ll}
\sigma_{l}=\operatorname{diag}\left(-I_{l}, I_{n-l},-I_{l}, I_{n-l}\right) & \text { for } l=1, \ldots, n-1 ; \\
c_{\lambda}=\operatorname{diag}\left(\lambda, I_{n-1}, \lambda^{-1}, I_{n-1}\right) & \text { for } \lambda^{2} \neq 0,1, \text { up to a sign, }
\end{array}
$$

and the pairs of $\mathrm{SO}_{2 n}(k)$-classes into which the $\mathrm{O}_{2 n}(k)$-class represented by $a_{\lambda}=$ $\operatorname{diag}\left(\lambda I_{n}, \lambda^{-1} I_{n}\right)$ splits, for $\lambda^{2} \neq 0,1$. The unipotent ones are those associated with the partitions

$$
\begin{array}{ll}
\left(2^{2 m}, 1^{2 n-4 m}\right) & \text { for } m=1, \ldots,[n / 2], \\
\left(3,2^{2 m}, 1^{2 n-3-4 m}\right) & \text { for } m=1, \ldots,[n / 2]-1
\end{array}
$$

and only $\left(2^{2(n / 2)}\right)$ for $n$ even corresponds to two distinct conjugacy classes. 
Proof. Semisimple classes. Let $s \in T$, and let $\Lambda$ be its set of eigenvalues. Adapting the analysis in type $C_{n}$ and replacing $s$ by a $\mathrm{GL}_{2 n}(k)$-conjugate if necessary, we see that if $s$ is spherical, then $|\Lambda| \leq 3$ and if $|\Lambda|=3$, then, up to a sign, $\Lambda=\left\{\lambda, \lambda^{-1}, 1\right\}$ for some $\lambda^{2} \neq 1$ and the multiplicity of $\lambda$ and $\lambda^{-1}$ is equal to 1 .

On the other hand, if $\Lambda=\left\{\lambda, \lambda^{-1}, 1\right\}$ with the multiplicity of $\lambda$ and $\lambda^{-1}$ equal to 1 , then $s$ is $\mathrm{GL}_{2 n}(k)$-conjugate to $c_{\lambda}=\operatorname{diag}\left(\lambda, I_{n-1}, \lambda^{-1}, I_{n-1}\right)$. Its centralizer $G_{c_{\lambda}}$ is equal to the identity component $H^{\circ}$ of the centralizer $H$ of the involution $\sigma_{1}=\operatorname{diag}\left(-1, I_{n-1},-1, I_{n-1}\right)$. By [Borel 1969, Proposition 9.1], we have $\mathrm{O}_{c_{\lambda}} \cong G / G_{c_{\lambda}}=G / H^{\circ}$. Since the index of $H^{\circ}$ in $H$ is finite, $O_{c_{\lambda}}$ is spherical if and only if $G / H \cong \mathrm{O}_{\sigma_{1}}$ is, and therefore $O_{c_{\lambda}}$ is spherical. The centralizer in $\mathrm{O}_{2 n}(k)$ of $c_{\lambda}$ contains the matrix

$$
M=\left(\begin{array}{cccc}
I_{n-1} & & \mathrm{O}_{n-1} & \\
& 0 & & 1 \\
\mathrm{O}_{n-1} & & I_{n-1} & \\
& 1 & & 0
\end{array}\right),
$$

so each $c_{\lambda}$ represents a single spherical $\mathrm{SO}_{2 n}(k)$-conjugacy class.

Let now $|\Lambda|=2$. If $\Lambda=\{ \pm 1\}$, then $s^{2}=1$ and $O_{s}$ is symmetric. The $\mathrm{GL}_{2 n}(k)$ class of $s$ is represented by $\sigma_{l}=\operatorname{diag}\left(-I_{l}, I_{n-l},-I_{l}, I_{n-l}\right)$ for some $l=1, \ldots, n-1$. The centralizer in $\mathrm{O}_{2 n}(k)$ of each $\sigma_{l}$ contains the matrix $M$ above described, so each $\sigma_{l}$ represents a single spherical $\mathrm{SO}_{2 n}(k)$-conjugacy class.

If $\Lambda=\left\{\lambda, \lambda^{-1}\right\}$ with $\lambda^{2} \neq 1$, we may assume that $s=a_{\lambda}=\operatorname{diag}\left(\lambda I_{n}, \lambda^{-1} I_{n}\right)$ whose centralizer is independent of $\lambda$ in the given range. Since $a_{\zeta}^{2} \in Z(G)$ for $\zeta$ a primitive fourth root of 1 , by Remark 2.4 all those classes are symmetric and hence spherical. The $\mathrm{O}_{2 n}(k)$-centralizer of $a_{\lambda}$ consists of all matrices $\operatorname{diag}\left(A,{ }^{t} A^{-1}\right)$ for some invertible $n \times n$ matrix $A$ and hence is contained in $\mathrm{SO}_{2 n}(k)$. Therefore the $\mathrm{O}_{2 n}(k)$-class of each $a_{\lambda}$ splits into two spherical $\mathrm{SO}_{2 n}(k)$-conjugacy classes.

Unipotent classes. By the discussion of $\mathrm{GL}_{2 n}(k)$-conjugacy, it suffices to consider a class for each admissible partition.

Let $u$ be a unipotent element in $G$, with associated partition $\underline{\lambda}$. Obtain $\underline{\mu}=$ $\left(\mu_{1}, \ldots, \mu_{l}\right)$ by taking a representative of each term occurring pairwise in $\underline{\lambda}$, and $\underline{\nu}=\left(v_{1}, \ldots, v_{m}\right)$ by taking the remaining distinct odd terms so that $2 n=2|\underline{\mu}|+|\underline{v}|$. A representative $u^{\prime}$ of $\mathrm{O}_{u}$ can be taken in the subgroup isomorphic to $\mathrm{SO}_{v_{1}+v_{2}}(k) \times$ $\cdots \times \mathrm{SO}_{v_{m-1}+v_{m}}(k) \times \mathrm{SO}_{2 \mu_{1}}(k) \times \cdots \times \mathrm{SO}_{2 \mu_{l}}(k)$ obtained by repeatedly immersing $\mathrm{SO}_{2 d_{1}}(k) \times \mathrm{SO}_{2 d_{2}}(k)$ into $\mathrm{SO}_{2\left(d_{1}+d_{2}\right)}(k)$ by

$$
\left(\left(\begin{array}{ll}
A_{1} & B_{1} \\
C_{1} & D_{1}
\end{array}\right),\left(\begin{array}{ll}
A_{2} & B_{2} \\
C_{2} & D_{2}
\end{array}\right)\right) \mapsto\left(\begin{array}{llll}
A_{1} & & B_{1} & \\
& A_{2} & & B_{2} \\
C_{1} & & D_{1} & \\
& C_{2} & & D_{2}
\end{array}\right)
$$


The component of $u^{\prime}$ in $\mathrm{SO}_{v_{i}+v_{i+1}}(k)$ is associated with the partition $\left(v_{i}, v_{i+1}\right)$, whereas the component of $u^{\prime}$ in $\mathrm{SO}_{2 \mu_{i}}(k)$ can be chosen to lie and be regular in the subgroup isomorphic to $\mathrm{SL}_{\mu_{i}}(k)$ obtained by the immersion $A \mapsto \operatorname{diag}\left(A,{ }^{t} A^{-1}\right)$. Thus $u^{\prime}$ lies in $\mathrm{SO}_{v_{1}+v_{2}}(k) \times \cdots \times \mathrm{SO}_{v_{m-1}+v_{m}}(k) \times \mathrm{SL}_{\mu_{1}}(k) \times \cdots \times \mathrm{SL}_{\mu_{l}}(k)$. A class in a semisimple group is spherical if and only if its projection onto each simple component is. By Remark 2.5 applied to $\mathrm{SL}_{\mu_{1}}(k) \times \cdots \times \mathrm{SL}_{\mu_{l}}(k)$, we see that if $u$ is spherical, then $\mu_{i} \leq 2$ for all $i$. We now show that under the same assumption, $v_{1} \leq 3$ so that $\underline{v}$ is either $(3,1)$ or the empty partition. It is enough to analyze the $\mathrm{SO}_{v_{1}+v_{2}}(k)$-class $\mathrm{O}$ of the component of $u^{\prime}$. Let $v_{1}=2 l+1$ and $v_{2}=2 j-1$ with $l \geq j \geq 1$, and let $\gamma_{1}, \ldots, \gamma_{l+j}$ be the simple roots of $\operatorname{SO}_{v_{1}+v_{2}}(k)$. The class $\mathrm{O}$ is represented by $x=\operatorname{diag}\left(A,{ }^{t} A^{-1}\right)\left(\begin{array}{ll}I & X \\ I\end{array}\right)$, where

$$
A=\left(\begin{array}{cccc}
1 & & & \\
1 & \ddots & & \\
& \ddots & \ddots & 1 \\
& & 1 & 1
\end{array}\right) \quad \text { and } \quad X=\left(\begin{array}{cccc}
0_{j-1} & & & \\
& 0 & 1 & \\
& -1 & 0 & \\
& & & 0_{l-1}
\end{array}\right)
$$

Since $\operatorname{diag}\left(A,{ }^{t} A^{-1}\right)$ lies in $X_{-\gamma_{1}}^{\prime} \cdots X_{-\gamma_{j+l-1}}^{\prime}$ and $\left(\begin{array}{ll}I & X \\ I\end{array}\right)$ lies in $B$, it follows from (1) and (2) that $x$ lies in a cell corresponding to an involution only if $j+l \leq 2$, whence the claim.

Conversely, let $\mathrm{O}_{u}$ be a unipotent class corresponding to $\left(2^{2 m}, 1^{2 n-4 m}\right)$ or to $\left(3,2^{2 m}, 1^{2 n-3-4 m}\right)$. Cantarini et al. [2005, Theorem 12, pages 37-38] give matrices that represent these classes also when $\operatorname{char}(k)$ is odd and their $B$-orbits satisfy the condition in Theorem 2.2.

Mixed classes. We show that there is no spherical element with Jordan decomposition $g=s u$ with $s \notin Z(G)$ and $u \neq 1$. We may assume that $s=c_{\lambda}, \sigma_{l}$, because $\operatorname{dim} B=\operatorname{dim} 0_{a_{\lambda}}<\operatorname{dim} 0_{a_{\lambda} u}$.

The subgroup $G_{c_{\lambda}}^{\circ}$ is of type $D_{n-1} \times k^{*}$ and is generated by $T$ and the root subgroups $X_{ \pm \alpha_{2}}, \ldots, X_{ \pm \alpha_{n}}$. The element $u \in G_{c_{\lambda}}^{\circ}$ corresponds to a partition $\underline{\pi}$ of $2 n-2$ from which we may construct, as before, the partitions $\underline{\mu}$ and $\underline{v}$. Since $u$ is spherical in $G_{c_{\lambda}}^{\circ}$, we have $\underline{\mu}=\left(2^{2 a}, 1^{2 b}\right)$ with $a$ possibly zero and $\underline{v}=(3,1)$ or trivial. The $G_{c_{\lambda}}$-class of $u$ may be represented by the element

$$
u^{\prime}=x_{\alpha_{2}}(1) x_{\alpha_{4}}(1) \cdots x_{\alpha_{2 a}}(1) x_{\alpha_{2 a+2}}(j) x_{\alpha_{2 a+2}+2 \alpha_{2 a+3}+\cdots+2 \alpha_{n-2}+\alpha_{n-1}+\alpha_{n}}(j) \text {, }
$$

where $j=1$ if $\underline{v}=(3,1)$ and $j=0$ if $\underline{v}$ is trivial. Then $c_{\lambda} u^{\prime}$ is regular in $\left\langle T, X_{ \pm \alpha_{1}}, X_{ \pm \alpha_{2 l}}, x_{\alpha_{2 a+2}}(j), x_{\alpha_{2 a+2}+2 \alpha_{2 a+3}+\cdots+2 \alpha_{n-2}+\alpha_{n-1}+\alpha_{n}}(j)\right\rangle_{l=1, \ldots, a}$. We may thus apply Lemma 2.8 to deduce that $s$ cannot be equal to $c_{\lambda}$.

Let then $s=\sigma_{l}$ for some $l$. The identity component of $G_{\sigma_{1}}$ is equal to $G_{c_{\lambda}}$, and we may use the argument above to show that $\sigma_{1} u$ cannot be spherical. Let $l \geq 2$. 
Then $G_{\sigma_{l}}^{\circ} \cong \mathrm{SO}_{2 l}(k) \times \mathrm{SO}_{2 n-2 l}(k)$ and it corresponds to the roots

$$
\alpha_{1}, \ldots, \alpha_{l-1}, \alpha_{l+1}+2 \alpha_{l+2}+\cdots+\alpha_{n-1}+\alpha_{n}, \alpha_{l+1}, \ldots, \alpha_{n} .
$$

Let $u=\left(u_{1}, u_{2}\right) \in \mathrm{SO}_{2 l}(k) \times \mathrm{SO}_{2 n-2 l}(k)=G_{\sigma_{l}}^{\circ}$. Since $G_{\sigma_{l} u}=G_{\sigma_{l}} \cap G_{u_{1}} \cap G_{u_{2}}$ is contained in $G_{\sigma_{l} u_{i}}$ for $i=1,2$, it is enough to show that $\sigma_{l} u_{i}$ is not spherical. We will do so for $u_{2}$, the other case being similar. Let $\underline{\lambda}$ be the partition associated with $u_{2}$, and let $\underline{\mu}$ and $\underline{v}$ be as above. We may find a representative $u_{2}^{\prime}$ in the $\mathrm{SO}_{2 n-2 l}(k)$-class of $u_{2}$ lying and being regular in a subgroup $H$ constructed as above for $s=c_{\lambda}$. If $u_{2} \neq 1$, the subgroup $H$ contains the root subgroups $X_{ \pm \alpha_{l+1}}$ and $\sigma_{l} u_{2}^{\prime}$ is regular in $H^{\prime}=\left\langle T, H, X_{ \pm \alpha_{l}}\right\rangle$. This proves the claim.

Type $\boldsymbol{B}_{n}$. Let $n \geq 2$. View $\mathrm{O}_{2 n+1}(k)$ as the subgroup of $\mathrm{GL}_{2 n+1}(k)$ of matrices preserving the bilinear form whose matrix is

$$
\left(\begin{array}{ccc}
1 & 0 & 0 \\
0 & 0 & I_{n} \\
0 & I_{n} & 0
\end{array}\right)
$$

in the canonical basis of $k^{2 n+1}$, so that $G=\mathrm{SO}_{2 n+1}(k)$ is the subgroup of such matrices with unit determinant. Fix $B$ to be the subgroup of matrices of the form

$$
\left(\begin{array}{ccc}
1 & 0 & { }^{t} \gamma \\
-A \gamma & A & A X \\
0 & 0 & { }^{t} A^{-1}
\end{array}\right)
$$

where $A$ is an invertible upper triangular matrix, $\gamma$ is a column in $k^{n}$ and the symmetric part of $X$ is $-(1 / 2) \gamma^{t} \gamma$. We fix $T \subset B$ as its subgroup of diagonal matrices.

We have $X_{\alpha_{i}}=\left\{I+t E_{i+1, i+2}-t E_{n+i+2, n+i+1}, t \in k\right\}$ for $i=1, \ldots, n-1$, $X_{\alpha_{n}}=\left\{I+t E_{1,2 n+1}-t E_{n+1,1}\right\}$, and $X_{-\alpha}={ }^{t} X_{\alpha}$ for every $\alpha \in \Phi$.

If $g, h \in G$ are $\mathrm{GL}_{2 n+1}(k)$-conjugate, then they are also $\mathrm{O}_{2 n+1}(k)$-conjugate by [Springer and Steinberg 1970, IV.2.15(ii)]. Thus, they are $G$-conjugate because $\mathrm{O}_{2 n+1}(k)=G \cup\left(-I_{2 n+1}\right) G$. Partitions in which even terms occur pairwise parametrize unipotent conjugacy classes in $G$ [Humphreys 1995, Section 7.11].

We shall frequently use the fact that the group $\mathrm{SO}_{2 n}(k)$ may be embedded into $G$ through the map $\imath$ defined by $X \mapsto \operatorname{diag}(1, X)$. Denote the image of $\imath$ by $K$.

Theorem 3.5. Let $G=\mathrm{SO}_{2 n+1}(k)$. The spherical semisimple classes in $G$ are represented by

$$
\begin{array}{ll}
\rho_{l} & =\operatorname{diag}\left(1,-I_{l}, I_{n-l},-I_{l}, I_{n-l}\right) \\
d_{\lambda}=\operatorname{diag}\left(1, \lambda, I_{n-1}, \lambda^{-1}, I_{n-1}\right), & \text { for } l=1, \ldots, n, \\
b_{\lambda}=\operatorname{diag}\left(1, \lambda I_{n}, \lambda^{-1} I_{n}\right) & \text { with } \lambda^{2} \neq 0,1 .
\end{array}
$$


The unipotent ones are those associated with $\left(2^{2 m}, 1^{2 n+1-4 m}\right)$ for $m=1, \ldots,[n / 2]$ and $\left(3,2^{2 m}, 1^{2 n-2-4 m}\right)$ for $m=1, \ldots,[(n-1) / 2]$. The spherical classes that are neither semisimple nor unipotent are represented by $\rho_{n} u$, where $u \in G_{\rho_{n}}^{\circ} \cong \mathrm{SO}_{2 n}(k)$ is a unipotent element associated with $\left(2^{2 m}, 1^{2 n-4 m}\right)$ for $m=1, \ldots,[n / 2]$.

Proof. Semisimple classes. Let $s \in T$ be a spherical element in $G$, and let $\Lambda$ be its set of eigenvalues. By the description of $T$, we always have $1 \in \Lambda$. By Lemma 2.6 applied to $K$ and Theorem 3.4, we see that $|\Lambda| \leq 4$. We claim that $|\Lambda|<4$. Assume that $|\Lambda|=4$. Then $-1 \in \Lambda$ and $s$ is conjugate to $s^{\prime}=\operatorname{diag}\left(1, \lambda,-1, t, \lambda^{-1},-1, t^{-1}\right)$ for some invertible diagonal submatrix $t$ and some scalar $\lambda$ with $\lambda^{2} \neq 1$. Thus $s^{\prime}$ is regular in $\left\langle T, X_{ \pm \alpha_{1}}, X_{ \pm\left(\alpha_{2}+\alpha_{3}+\cdots+\alpha_{n}\right)}\right\rangle$ whose semisimple part is of type $B_{2}$, and by Lemma 2.8 we have the claim. It follows that $|\Lambda|=2,3$. If $|\Lambda|=2$, the element $s$ is conjugate to some involution $\rho_{l}=\operatorname{diag}\left(1,-I_{l}, I_{n-l},-I_{l}, I_{n-l}\right)$ for some $l=1, \ldots, n$; hence it is spherical. If $|\Lambda|=3$, then $\Lambda=\left\{1, \lambda, \lambda^{-1}\right\}$ and the multiplicities of $\lambda$ and 1 cannot be both greater than 1, by Lemma 2.6 applied to $K$ and the discussion in Theorem 3.4 for spherical semisimple elements. Thus, $s$ is conjugate either to $b_{\lambda}=\operatorname{diag}\left(1, \lambda I_{n}, \lambda^{-1} I_{n}\right)$ or to $d_{\lambda}=\operatorname{diag}\left(1, \lambda, I_{n-1}, \lambda^{-1}, I_{n-1}\right)$, for $\lambda^{2} \neq 1,0$. A representative of $\mathrm{O}_{b_{\lambda}}$ satisfying the condition in Theorem 2.2 is found in [Cantarini et al. 2005, Theorem 15, page 44] and it is well defined in odd characteristic too, so $b_{\lambda}$ is indeed spherical. Moreover, $G_{d_{\lambda}}=G_{\rho_{1}}^{\circ}$. Hence $d_{\lambda}$ is also spherical because $\rho_{1}$ is and the index of $G_{\rho_{1}}^{\circ}$ in $G_{\rho_{1}}$ is finite.

Unipotent classes. Let $u$ be a spherical unipotent element in $G$ associated with the partition $\underline{\lambda}$. Let $\underline{\mu}$ and $\underline{v}$ be constructed as in Theorem 3.4 with $2 n+1=$ $2|\mu|+|\underline{v}|$. We may find a representative $u^{\prime}$ of $\mathrm{O}_{u}$ in a subgroup isomorphic to $\mathrm{SO}_{v_{1}}(k) \times \mathrm{SO}_{v_{2}+v_{3}}(k) \times \cdots \times \mathrm{SO}_{v_{m-1}+v_{m}}(k) \times \mathrm{SO}_{2 \mu_{1}}(k) \times \cdots \times \mathrm{SO}_{2 \mu_{l}}(k)$. Such a subgroup can be obtained using the embeddings in the proof of Theorem 3.4 and the embedding of $\mathrm{SO}_{2 d_{1}+1}(k) \times \mathrm{SO}_{2 d_{2}}(k)$ into $\mathrm{SO}_{2\left(d_{1}+d_{2}\right)+1}(k)$ given by

$$
\left(\left(\begin{array}{ccc}
1 & \alpha_{1} & \beta_{1} \\
\gamma_{1} & A_{1} & B_{1} \\
\delta_{1} & C_{1} & D_{1}
\end{array}\right),\left(\begin{array}{ll}
A_{2} & B_{2} \\
C_{2} & D_{2}
\end{array}\right)\right) \mapsto\left(\begin{array}{ccccc}
1 & \alpha_{1} & & \beta_{1} & \\
\gamma_{1} & A_{1} & & B_{1} & \\
& & A_{2} & & B_{2} \\
\delta_{1} & C_{1} & & D_{1} & \\
& & C_{2} & & D_{2}
\end{array}\right) .
$$

The component of $u^{\prime}$ in $\mathrm{SO}_{v_{1}}(k)$ corresponds to $\left(v_{1}\right)$, so it is regular therein. Hence, its $\mathrm{SO}_{v_{1}}(k)$-class is spherical only if $v_{1} \leq 3$. Therefore $\underline{v}=(3)$ or $\underline{v}=(1)$. Moreover, as in Theorem 3.4, the component in $\mathrm{SO}_{2 \mu_{j}}(k)$ can be chosen to lie and be regular in a subgroup isomorphic to $\mathrm{SL}_{\mu_{j}}(k)$, forcing $\mu_{i} \leq 2$ for every $i$. Conversely, for a unipotent class associated with $\left(2^{m}, 1^{2 n+1-4 m}\right)$ or $\left(3,2^{2 m}, 1^{2 n-4 m-2}\right)$, the representatives in [Cantarini et al. 2005, Theorem 12, pages 38-39] are well defined in odd characteristic, and the corresponding $B$-orbits satisfy the condition in Theorem 2.2. 
Mixed classes. Let $g=s u$ be the Jordan decomposition of a spherical element in $G$ with $s, u \neq 1$. If $s=b_{\lambda}$ for some $\lambda$, then $u$ and $b_{\lambda}$ lie in $G_{b_{\lambda}} \subset K$. By Lemma 2.6 the element $s u$ would be spherical in $K$, but this is excluded by Theorem 3.4.

If $s=\rho_{l}$ for some $l$, its centralizer is isomorphic to $\mathrm{SO}_{2 l}(k) \times \mathrm{SO}_{2 n-2 l+1}(k)$ and it corresponds to the roots $\alpha_{1}, \ldots, \alpha_{l-1}, \alpha_{l}+2 \alpha_{l+1}+\cdots+2 \alpha_{n}, \alpha_{l+1}, \ldots, \alpha_{n}$. Then $u=\left(u_{1}, u_{2}\right) \in \mathrm{SO}_{2 l}(k) \times \mathrm{SO}_{2 n-2 l+1}(k)$.

First assume that $u_{2}$ corresponds to the partition $\left(3,2^{2 a}\right)$ of $2 n-2 l+1$, so that $n-l$ is odd. We claim that $\rho_{l} u_{2}$ is not spherical. Then, since $G_{\rho_{l} u} \subset G_{\rho_{l} u_{2}}$, we may conclude that the class $0_{\rho_{l} u}$ cannot be spherical in this case. The $\mathrm{SO}_{2 n-2 l+1}(k)$ class of $u_{2}$ may be represented by the element $u_{2}^{\prime}=x_{\alpha_{l+1}}(1) x_{\alpha_{l+3}}(1) \cdots x_{\alpha_{n}}$ (1) so

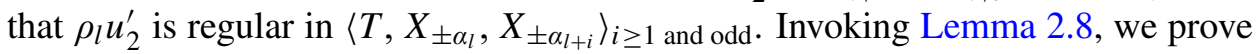
the claim.

If $u_{2}$ does not correspond to the partition $\left(3,2^{2 a}\right)$, we may find a representative of $\mathrm{O}_{\rho_{l} u}$ that lies in $K$. By Lemma 2.6 and Theorem 3.4, this is possible only if $\rho_{l}=\imath(t)$ for some $t \in Z(K)$. Therefore $g=\rho_{n} \mathrm{v}=\operatorname{diag}\left(1,-I_{2 n}\right) \mathrm{v}$ for some spherical unipotent $\mathrm{v}$ in $G_{\rho_{n}}^{\circ}=K$. We claim that the partition $\underline{\lambda}$ of $2 n$ associated with $\mathrm{v}$ has no term equal to 3 . If $\underline{\lambda}=\left(3,2^{2 a}, 1^{c}\right)$, the $K$-class of $\mathrm{v}$ could be represented by $\mathrm{v}^{\prime}=x_{\alpha_{1}}(1) x_{\alpha_{3}}(1) \cdots x_{\alpha_{2 a-1}}(1) x_{\alpha_{n-1}}(1) x_{\alpha_{n-1}+2 \alpha_{n}}(1)$. The element $\rho_{l} \mathbf{v}^{\prime}$ is regular in $\left\langle T, X_{ \pm \alpha_{1}}, X_{ \pm \alpha_{3}}, \ldots X_{ \pm \alpha_{2 a-1}}, X_{ \pm \alpha_{n-1}}, X_{ \pm \alpha_{n}}\right\rangle$, whose semisimple part is of type $a A_{1} \times B_{2}$; hence the claim follows from Lemma 2.8. Conversely, let $g=\rho_{n} u$ with $u$ corresponding to $\left(2^{2 m}, 1^{2 n-4 m}\right)$ for some $m$. The representative of its class provided in [Cantarini et al. 2005, Theorem 21, page 52] is well defined in odd characteristic and it allows application of Theorem 2.2.

Finally assume that $s=d_{\lambda}$ for some $\lambda$. Then $u \in G_{d_{\lambda}}=G_{\rho_{1}}^{\circ}$ and we may apply the arguments used for $s=\rho_{1}$ to show that $s u$ cannot be spherical.

\section{Type $E_{6}$.}

Theorem 3.6. Let $G$ be simply-connected of type $E_{6}$. The spherical classes in $G$ are either semisimple or unipotent up to a central element. The semisimple ones are symmetric and up to a central factor are represented by $p_{1}=h_{1}(-1) h_{4}(-1) h_{6}(-1)$ and $p_{2, c}=h_{1}\left(c^{2}\right) h_{2}\left(c^{3}\right) h_{3}\left(c^{4}\right) h_{4}\left(c^{6}\right) h_{5}\left(c^{5}\right) h_{6}\left(c^{4}\right)$ for $c \in k$ with $c^{3} \neq 1,0$. The unipotent ones are those of type $A_{1}, 2 A_{1}$ and $3 A_{1}$.

Proof. Semisimple classes. Let $s \in T$ be spherical. We may apply [Humphreys 1995, Theorem 2.15] to choose $s$ so that $G_{s}$ is generated by $T$ and $X_{ \pm \alpha}$ for $\alpha$ in a subsystem $\Phi(\Pi) \subset \Phi$ with basis a subset $\Pi$ of $\Delta \cup\left\{-\beta_{1}\right\}$. By Theorem 2.2 we have $\operatorname{dim} O_{s} \leq \ell\left(w_{0}\right)+\operatorname{rk}\left(1-w_{0}\right)$ and a dimension count shows that $\Pi$ can only be one of the following subsets:

$$
\begin{array}{ll}
\Pi_{1}=\left\{\alpha_{1}, \alpha_{3}, \alpha_{4}, \alpha_{5}, \alpha_{6},-\beta_{1}\right\}, & \Pi_{2}=\left\{\alpha_{1}, \alpha_{2}, \alpha_{4}, \alpha_{5}, \alpha_{6},-\beta_{1}\right\}, \\
\Pi_{3}=\left\{\alpha_{1}, \alpha_{2}, \alpha_{3}, \alpha_{4}, \alpha_{6},-\beta_{1}\right\} & \text { of type } A_{5} \times A_{1},
\end{array}
$$


or

$$
\begin{array}{ll}
\Pi_{4}=\left\{\alpha_{1}, \alpha_{2}, \alpha_{3}, \alpha_{4}, \alpha_{5}\right\}, & \Pi_{5}=\left\{\alpha_{2}, \alpha_{3}, \alpha_{4}, \alpha_{5},-\beta_{1}\right\} \\
\Pi_{6}=\left\{\alpha_{2}, \alpha_{3}, \alpha_{4}, \alpha_{5}, \alpha_{6}\right\} & \text { of type } D_{5} .
\end{array}
$$

Let us put $H_{i}=\left\langle T, X_{ \pm \alpha}, \alpha \in \Pi_{i}\right\rangle$ for $i=1, \ldots, 6$. The sets $\Pi_{i}$ for $i=1,2,3$ are $\mathbb{R}$-bases for the span of $\Delta$, and one may find automorphisms of $\Phi$ mapping $\Pi_{i}$ for $i=2,3$ to $\pm \Pi_{1}$. On the other hand, $\operatorname{Aut}(\Phi)=\left\{-w_{0}\right\} \ltimes W$ so any element $s$ whose centralizer is $H_{2}$ or $H_{3}$ is $N(T)$-conjugate to an element whose centralizer is $H_{1}$. The elements $s$ for which $G_{s}=H_{1}$ are $p_{1}=h_{1}(-1) h_{4}(-1) h_{6}(-1)$ and $z p_{1}$ for any $z \in Z(G)$. Conjugation by these elements is an involution, so $0_{p_{1}}$ is symmetric. This completes the analysis for $\Pi_{i}$ with $i \leq 3$.

The subgroups $H_{4}$ and $H_{6}$ are $\dot{w}_{0}$-conjugate, so any element whose centralizer is $H_{4}$ is $N(T)$-conjugate to an element whose centralizer is $H_{6}$. Besides, the automorphism of $\Phi$ defined by $\alpha_{1} \mapsto-\beta_{1}, \alpha_{2} \mapsto \alpha_{3}, \alpha_{3} \mapsto \alpha_{2}, \alpha_{j} \mapsto \alpha_{j}$ for $j=4,5,6$ maps $\Pi_{4}$ onto $\Pi_{5}$. As before, we may conclude that $H_{5}$ is $N(T)$ conjugate to $H_{4}$ and any element whose centralizer is $H_{5}$ is $N(T)$-conjugate to an element whose centralizer is $H_{4}$. The elements whose centralizer is $H_{4}$ are $p_{2, c}=h_{1}\left(c^{2}\right) h_{2}\left(c^{3}\right) h_{3}\left(c^{4}\right) h_{4}\left(c^{6}\right) h_{5}\left(c^{5}\right) h_{6}\left(c^{4}\right)$ for $c \in k$ with $c^{3} \neq 1,0$. Multiplying $c$ by a third root of unity yields the same element multiplied by a central one. Since $p_{2,-1}$ is an involution, $\mathrm{O}_{p_{2, c}}$ is spherical by Remark 2.4 . We claim that $p_{2, c}$ is not conjugate to $p_{2, d}$ for $c \neq d$. If they were $G$-conjugate, they would be $N(T)$ conjugate by [Springer and Steinberg 1970, Section 3.1], so there would exist a $\sigma \in W$ such that $\dot{\sigma} p_{2, c} \dot{\sigma}^{-1}=p_{2, d}$. Thus, $\sigma$ would stabilize $\Phi\left(\Pi_{4}\right)$ and would restrict to an automorphism of $\Phi\left(\Pi_{4}\right)$. Its restriction would therefore be of the form $\tau w$, where $\tau$ acts an automorphism of the Dynkin diagram of type $D_{5}$ and $w$ lies in the Weyl group $W^{\prime}$ of $H_{4}$, which is contained in $W$. Then $\sigma w^{-1}$ would lie in $W$, and it would act on $\Pi_{4}$ as $\tau$. Besides, two automorphisms $\psi_{1}, \psi_{2}$ of $\Phi$ coinciding on $\Pi_{4}$ are equal. Indeed, for $\alpha=\psi_{1} \psi_{2}^{-1}\left(\alpha_{6}\right)$, we have $\left\langle\alpha_{j}, \alpha\right\rangle=0$ for $j=1,2,3,4$ and $\left\langle\alpha, \alpha_{5}\right\rangle=-1$. Such a root $\alpha$ can only be $\alpha_{6}$, so $\psi_{1} \psi_{2}^{-1}=1$. It follows that $\sigma w^{-1}$ is either the identity, when $\tau=1$, or it is the automorphism mapping $\alpha_{j}$ to $\alpha_{j}$ for $j=1,3,4$, interchanges $\alpha_{2}$ and $\alpha_{5}$, and maps $\alpha_{6}$ to $-\beta_{1}$. However, one may verify that the second possibility cannot happen because such an automorphism is equal to $s_{1} s_{3} s_{4} s_{5} s_{2} s_{4} s_{6} s_{5} s_{3} s_{4} s_{1} s_{3} s_{2} s_{4} s_{5} s_{6}\left(-w_{0}\right)$; hence it does not lie in $W$. Therefore $\tau=1$ and $\sigma=w \in W^{\prime}$. Since $G_{p_{2, c}}=H_{4}$, conjugation by the lift in $N(T)$ of an element in $W^{\prime}$ does not modify $p_{2, c}$, so $p_{2, c}$ and $p_{2, d}$ represent distinct classes.

Unipotent classes. Let $O$ be a nontrivial spherical unipotent class. Then $\operatorname{dim} O \leq$ $\ell\left(w_{0}\right)+\operatorname{rk}\left(1-w_{0}\right)$ by Theorem 2.2 , so 0 is of type $A_{1}, 2 A_{1}$ or $3 A_{1}$. Conversely, the arguments in [Cantarini et al. 2005, Theorem 13, pages 39-40] apply in good 
characteristic and show that the listed orbits have a representative whose $B$-orbit satisfies the conditions of Theorem 2.2.

Mixed classes. A dimension counting together with Lemma 2.7 shows that no class $\mathcal{O}_{s u}$ with $s \notin Z(G)$ and $u \neq 1$ can be spherical.

\section{Type $E_{7}$.}

Theorem 3.7. Let $G$ be simply-connected of type $E_{7}$. The spherical classes in $G$ are either semisimple or unipotent up to a central element. The semisimple ones are symmetric and are represented by $q_{1}=h_{2}(\zeta) h_{5}(-\zeta) h_{6}(-1) h_{7}(\zeta)$, where $\zeta$ is a fixed primitive fourth root of $1 ; q_{2}=h_{3}(-1) h_{5}(-1) h_{7}(-1) ; z q_{1}$, and $z q_{2}$ for $z \in Z(G) ;$ and $q_{3, a}=h_{1}\left(a^{2}\right) h_{2}\left(a^{3}\right) h_{3}\left(a^{4}\right) h_{4}\left(a^{6}\right) h_{5}\left(a^{5}\right) h_{6}\left(a^{4}\right) h_{7}\left(a^{3}\right)$ with $a^{2} \neq 1,0$. The unipotent ones are those of type $A_{1}, 2 A_{1},\left(3 A_{1}\right)^{\prime},\left(3 A_{1}\right)^{\prime \prime}$ and $4 A_{1}$.

Proof. Semisimple classes. Let $s \in T$ be a spherical element. Proceeding as we did in Theorem 3.6, using that $\operatorname{dim} O_{s} \leq \operatorname{dim} B$, we may choose $s$ so that $G_{s}$ is generated by $T$ and $X_{ \pm \alpha}$ for $\alpha \in \Phi(\Pi)$ where $\Pi$ is one of the following subsets of $\Delta \cup\left\{-\beta_{1}\right\}$ :

$$
\begin{array}{ll}
\Pi_{1}=\left\{\alpha_{1}, \alpha_{3}, \alpha_{4}, \alpha_{5}, \alpha_{6}, \alpha_{7},-\beta_{1}\right\} & \text { of type } A_{7} ; \\
\Pi_{2}=\left\{\alpha_{2}, \alpha_{3}, \alpha_{4}, \alpha_{5}, \alpha_{6}, \alpha_{7},-\beta_{1}\right\}, & \\
\Pi_{3}=\left\{\alpha_{1}, \alpha_{2}, \alpha_{3}, \alpha_{4}, \alpha_{5}, \alpha_{7},-\beta_{1}\right\} & \text { of type } D_{6} \times A_{1} ; \\
\Pi_{4}=\left\{\alpha_{1}, \alpha_{2}, \alpha_{3}, \alpha_{4}, \alpha_{5}, \alpha_{6}\right\} & \text { of type } E_{6} .
\end{array}
$$

Let us put $H_{i}=\left\langle T, X_{ \pm \alpha}, \alpha \in \Pi_{i}\right\rangle$.

There is only one element, up to a central one, whose centralizer is $H_{1}$, and this is $q_{1}=h_{2}(\zeta) h_{5}(-\zeta) h_{6}(-1) h_{7}(\zeta)$, where $\zeta$ is a fixed primitive fourth root of 1 . Since $q_{1}^{2}=h_{2}(-1) h_{5}(-1) h_{7}(-1) \in Z(G)$, the corresponding class is symmetric by Remark 2.4. The root systems generated by $\Pi_{2}$ and $\Pi_{3}$ are mapped onto each other by elements in $\operatorname{Aut}(\Phi)=W$. Thus, each element whose centralizer is $\mathrm{H}_{2}$ is $N(T)$-conjugate to one whose centralizer is $H_{3}$, and it is enough to look at $\Pi_{2}$. The elements whose centralizer is $H_{2}$ are $q_{2}=h_{3}(-1) h_{5}(-1) h_{7}(-1)$ and $z q_{2}$ for $z \in Z(G)$. The corresponding classes are symmetric. The elements whose centralizer is $H_{4}$ are $q_{3, a}=h_{1}\left(a^{2}\right) h_{2}\left(a^{3}\right) h_{3}\left(a^{4}\right) h_{4}\left(a^{6}\right) h_{5}\left(a^{5}\right) h_{6}\left(a^{4}\right) h_{7}\left(a^{3}\right)$ for $a^{2} \neq 1,0$. For $\xi$ a primitive fourth root of unity, we have $q_{3, \xi}^{2} \in Z(G)$ and hence all such classes are symmetric. Multiplication of $q_{3, a}$ by the nontrivial central element gives $q_{3,-a}$. We claim that $q_{3, a}$ is never conjugate to $q_{3, b}$ for $a \neq b$. If they were $G$-conjugate, they would be $N(T)$-conjugate, so there would exist a $\sigma \in W$ for which $\dot{\sigma} q_{3, a} \dot{\sigma}^{-1}=q_{3, b}$. Such a $\sigma$ would preserve $\Phi\left(\Pi_{4}\right)$, and its restriction to it would be an automorphism. As in the proof of Theorem 3.6, we see that for some $w$ in the Weyl group $W^{\prime}$ of $H_{4}$, the restriction to $\Phi\left(\Pi_{4}\right)$ of $\sigma w^{-1} \in W$ would come from an automorphism of the Dynkin diagram of type $E_{6}$. There is no 
automorphism of $\Phi$ whose restriction to $E_{6}$ is the nontrivial automorphism. Indeed, if such an automorphism $\tau$ existed, for $\alpha=\tau\left(\alpha_{7}\right)$ we would have $\left\langle\alpha, \alpha_{j}\right\rangle=0$ for $j=2,3,4,5,6$ and $\left\langle\alpha, \alpha_{1}\right\rangle=-1$, but there is no such $\alpha \in \Phi$. Therefore $\sigma w^{-1}$ is the identity on $\Phi\left(\Pi_{4}\right)$. By uniqueness of the extension of an automorphism from $E_{6}$ to $E_{7}$ we have $\sigma=w \in W^{\prime}$. Since $G_{q_{3, a}}=H_{4}$, conjugation by lifts in $N(T)$ of elements in $W^{\prime}$ preserves $q_{3, a}$.

Unipotent classes. Let $u \neq 1$ be a spherical unipotent element. Then $\operatorname{dim} \mathrm{O}_{u} \leq$ $\operatorname{dim} B$, so $O_{u}$ is either of type $r A_{1}$ for some $r$, or of type $A_{2}$. In the latter case, $u$ would be regular in a Levi subgroup of type $A_{2}$, so this case cannot occur by Remark 2.5. The arguments in [Cantarini et al. 2005, Theorem 13, pages 39-40] apply also in good characteristic and show that for all unipotent classes of type $r A_{1}$, there is a representative whose $B$-orbit satisfies the condition in Theorem 2.2.

Mixed classes. We claim that there is no spherical element with Jordan decomposition $g=s u$ with $s \notin Z(G)$ and $u \neq 1$. Indeed, $O_{s}$ would be spherical and $u$ would be spherical in $G_{s}^{\circ}$. A dimensional argument shows that this is possible only if $s \in 0_{q_{2}}$ up to a central element and $u$ is nontrivial only in the component of type $A_{1}$ in $G_{s}$. It follows from the discussion of semisimple elements that we may choose $s$ so that $G_{s}=H_{3}$ with notation as before, so that we may choose $g$ to be conjugate to $s x_{-\alpha_{7}}(1)$. Conjugation of $g$ by $x_{-\alpha_{6}}(1)$ and Chevalley's commutator formula would give $z=s x_{-\alpha_{6}}(a) x_{-\alpha_{7}}(1) x_{-\alpha_{6}-\alpha_{7}}(b) \in \mathfrak{O}_{g}$ for some nonzero $a, b \in k$. Conjugating $z$ by a suitable element in $X_{-\alpha_{6}-\alpha_{7}}^{\prime}$, we could get rid of the term in $X_{-\alpha_{6}-\alpha_{7}}^{\prime}$, obtaining an element in $\mathrm{O}_{g} \cap B s_{6} s_{7} B$. By Theorem 2.3, the class $O_{g}$ cannot be spherical.

\section{Type $E_{8}$.}

Theorem 3.8. Let $G$ be of type $E_{8}$. The spherical classes are either semisimple or unipotent. The semisimple ones are symmetric, and they are represented by $r_{1}=h_{2}(-1) h_{3}(-1)$ and $r_{2}=h_{2}(-1) h_{5}(-1) h_{7}(-1)$. The unipotent ones are those of type $A_{1}, 2 A_{1}, 3 A_{1}$ and $4 A_{1}$.

Proof. Semisimple classes. Let $s \in T$ be a spherical element. Proceeding as we did in Theorems 3.6 and 3.7 we see that, up to $N(T)$-conjugation, the centralizer $G_{s}$ is generated by $T$ and by the $X_{ \pm \alpha}$ for $\alpha$ in a subsystem with basis either $\left\{\alpha_{2}, \alpha_{3}, \alpha_{4}, \alpha_{5}, \alpha_{6}, \alpha_{7}, \alpha_{8},-\beta_{1}\right\}$ of type $D_{8}$ or $\left\{\alpha_{1}, \alpha_{2}, \alpha_{3}, \alpha_{4}, \alpha_{5}, \alpha_{6}, \alpha_{7},-\beta_{1}\right\}$ of type $E_{7} \times A_{1}$. Then $s$ is conjugate either to $r_{1}=h_{2}(-1) h_{3}(-1)$ or to $r_{2}=$ $h_{2}(-1) h_{5}(-1) h_{7}(-1)$. Since $r_{1}^{2}=r_{2}^{2}=1$ the corresponding classes are symmetric.

Unipotent classes. Let $O$ be a nontrivial spherical unipotent class. Then $\operatorname{dim} O \leq$ $\operatorname{dim} B$, so $O$ is either of type $r A_{1}$ for some $r$, or it is of type $A_{2}$. The latter case is excluded as in the case of $G$ of type $E_{7}$. Conversely, the arguments in [Cantarini et al. 2005, Theorem 13, pages 39-40] apply in good characteristic and show that 
for each orbit of type $r A_{1}$ in $G$, we may find a representative whose $B$-orbit satisfies the condition in Theorem 2.2.

Mixed classes. We claim that there is no spherical element with Jordan decomposition $g=s u$ with $s, u \neq 1$. Indeed, by dimensional reasons, $s$ would be conjugate to $r_{2}$ and $u$ would lie in the component of type $A_{1}$ in $G_{r_{2}}=\left\langle T, X_{ \pm \beta_{1}}, X_{ \pm \alpha_{i}}\right\rangle_{i=1, \ldots, 7}$. In other words, we could assume $g=r_{2} x_{-\beta_{1}}(1)$. Let $\gamma=\beta_{1}-\alpha_{8}$. Conjugation of $g$ by $\dot{s}_{\gamma}$ gives $t x_{-\alpha_{8}}(a) \in \mathbb{O}_{g}$ for some nonzero $a \in k$ and some $t \in T$. Since $r_{2}$ does not commute with $X_{ \pm\left(\beta_{1}-\alpha_{8}-\alpha_{7}\right)}^{\prime}$ and $s_{\gamma}\left(\alpha_{7}+\alpha_{8}-\beta_{1}\right)=\alpha_{7}$, the element $t$ does not commute with $X_{ \pm \alpha_{7}}^{\prime}$. Since $s_{\gamma}\left(\alpha_{7}+\alpha_{8}\right)=\alpha_{7}+\alpha_{8}$ and $r_{2}$ does not commute with $X_{ \pm\left(\alpha_{7}+\alpha_{8}\right)}^{\prime}$, the same holds for $t$. Then conjugation of $t x_{-\alpha_{8}}(a)$ by $x_{-\alpha_{7}}(1)$ would give $t x_{-\alpha_{7}}(b) x_{-\alpha_{8}}(a) x_{-\alpha_{7}-\alpha_{8}}(c) \in \mathbb{O}_{g}$ for some nonzero $b, c \in k$. Conjugation by a suitable element in $X_{-\alpha_{7}-\alpha_{8}}^{\prime}$ would yield an element $x \in \mathrm{O}_{g} \cap T X_{-\alpha_{7}}^{\prime} X_{-\alpha_{8}}^{\prime}$. By (1) and (2), $x$ would lie in $\mathcal{O}_{g} \cap B s_{7} s_{8} B$, leading to a contradiction.

\section{Type $F_{4}$.}

Theorem 3.9. Let $G$ be of type $F_{4}$. The spherical semisimple classes are symmetric and represented by $f_{1}=h_{\alpha_{2}}(-1) h_{\alpha_{4}}(-1)$ and $f_{2}=h_{\alpha_{3}}(-1)$. The spherical unipotent ones are those of type $r A_{1}+s \tilde{A}_{1}$ for $r, s \in\{0,1\}$. There is a spherical class that is neither semisimple nor unipotent, and it is represented by $f_{2} x_{\beta_{1}}(1)$.

Proof. Semisimple classes. Let $s \in T$ be a spherical element in $G$. A dimension counting similar to the previous exceptional cases shows that $G_{s}$ is $N(T)$-conjugate to the subgroup generated by $T$ and the root subgroups corresponding to roots in a subsystem with basis either $\Pi_{1}=\left\{-\beta_{1}, \alpha_{2}, \alpha_{3}, \alpha_{4}\right\}$ or $\Pi_{2}=\left\{\alpha_{1}, \alpha_{2}, \alpha_{3},-\beta_{1}\right\}$. They correspond to the involutions $f_{1}=h_{\alpha_{2}}(-1) h_{\alpha_{4}}(-1)$ and $f_{2}=h_{\alpha_{3}}(-1)$, respectively, which are indeed spherical.

Unipotent classes. Let $O$ be a nontrivial spherical unipotent class in $G$. Then $\operatorname{dim} 0 \leq \operatorname{dim} B$, so $O$ is either of type $A_{1}, \tilde{A}_{1}$ or $A_{1}+\tilde{A}_{1}$. Conversely, the arguments in [Cantarini et al. 2005, Theorem 13, pages 39-40] hold in good characteristic and show that Theorem 2.2 applies to these three classes.

Mixed classes. Let $g=s u$ be the Jordan decomposition of a spherical element with $s, u \neq 1$. Since $\operatorname{dim} 0_{f_{1}}=\operatorname{dim} B$, we may assume $s=f_{2}$. Also, $G_{f_{2}}$ is a reductive group of type $B_{4}$. A dimensional argument shows that $u$ lies in the minimal unipotent class in $G_{f_{2}}$, so we may assume $g=f_{2} x_{-\left(2 \alpha_{1}+3 \alpha_{2}+4 \alpha_{3}+2 \alpha_{4}\right)}(1)=f_{2} x_{-\beta_{1}}(1)$. We have $\operatorname{dim} O_{g}=\operatorname{dim} B$. The proof in [Cantarini et al. 2005, Theorem 23] contains an incorrect argument, which we rectify here.

The element $f_{2}=h_{\alpha_{3}}(-1)$ lies in the subgroup $G_{1}=\left\langle X_{ \pm \alpha_{i}}, i=2,3,4\right\rangle$ of type $C_{3}$. By looking at the centralizer of $f_{2}$ in $G_{1}$ we see that, up to an element in $Z\left(G_{1}\right)$, the $G_{1}$-conjugacy class of $f_{2}$ is represented by $\sigma_{1}$ with notation as in Theorem 3.3. By [Cantarini et al. 2005, Theorem 15, page 42], the $G_{1}$-class of $\sigma_{1}$ 
has a representative in $s_{4} s_{\alpha_{2}+2 \alpha_{3}+\alpha_{4}} T$ when $k=\mathbb{C}$. The same matrix represents the class in good characteristic. Besides, $G_{1}$ centralizes $X_{ \pm \beta_{1}}$, so $f_{2} x_{-\beta_{1}}(1)$ can be represented by an element $z \in s_{4} s_{\alpha_{2}+2 \alpha_{3}+\alpha_{4}} T X_{-\beta_{1}}^{\prime} \subset s_{4} s_{\alpha_{2}+2 \alpha_{3}+\alpha_{4}} T X_{\beta_{1}}^{\prime} s_{\beta_{1}} X_{\beta_{1}}^{\prime} \subset$ $X_{\beta_{1}}^{\prime} w_{0} s_{2} T X_{\beta_{1}}^{\prime}$. Conjugating $z$ by $\dot{s}_{2} \dot{s}_{1}$, we obtain an element $z^{\prime} \in B w_{0} s_{1} B \cap O_{g}$. Thus, $w_{\mathscr{O}_{g}} \geq w_{0} s_{2}$ and $w_{\mathscr{O}_{g}} \geq w_{0} s_{1}$, forcing $w_{0}=w_{\mathscr{O}_{g}}$ (notation as in Section 2). Then $O_{g}$ has a representative whose $B$-orbit satisfies the condition in Theorem 2.2 and therefore is spherical.

\section{Acknowledgments}

I thank Mauro Costantini for pointing out an incorrect argument in the discussion of type $F_{4}$ in [Cantarini et al. 2005] and in a previous version of this manuscript. It is corrected in Theorem 3.9. I am grateful to the referee for suggesting a significant reordering of the exposition.

\section{References}

[Borel 1969] A. Borel, Linear algebraic groups, W. A. Benjamin, New York-Amsterdam, 1969. MR 40 \#4273 Zbl 0186.33201

[Bourbaki 1981] N. Bourbaki, Éléments de mathématique: Groupes et algèbres de Lie, Chapitres 4, 5 et 6, Masson, Paris, 1981. MR 83g:17001 Zbl 0483.22001

[Brion 1986] M. Brion, "Quelques propriétés des espaces homogènes sphériques", Manuscripta Math. 55:2 (1986), 191-198. MR 87g:14054 Zbl 0604.14048

[Cantarini et al. 2005] N. Cantarini, G. Carnovale, and M. Costantini, "Spherical orbits and representations of $U_{\epsilon}(\mathfrak{g})$ ", Transform. Groups 10:1 (2005), 29-62. MR 2005m:17020 Zbl 1101.17006

[Carnovale 2008] G. Carnovale, "Spherical conjugacy classes and involutions in the Weyl group", Math. Z. 260:1 (2008), 1-23. MR 2009d:20091 Zbl 1145.14040

[Carnovale 2009] G. Carnovale, "Spherical conjugacy classes and Bruhat decomposition", Ann. Inst. Fourier (Grenoble) 59:6 (2009), 2329-2357.

[Carter 1985] R. W. Carter, Finite groups of Lie type: Conjugacy classes and complex characters, Wiley, New York, 1985. MR 87d:20060 Zbl 0567.20023

[Ellers and Gordeev 2004] E. W. Ellers and N. Gordeev, "Intersection of conjugacy classes with Bruhat cells in Chevalley groups", Pacific J. Math. 214:2 (2004), 245-261. MR 2004m:20091 Zbl 1062.20050

[Ellers and Gordeev 2007] E. W. Ellers and N. Gordeev, "Intersection of conjugacy classes with Bruhat cells in Chevalley groups: The cases $\mathrm{SL}_{n}(K), \mathrm{GL}_{n}(K)$ ", J. Pure Appl. Algebra 209:3 (2007), 703-723. MR 2007m:20071 Zbl 1128.20034

[Fowler and Röhrle 2008] R. Fowler and G. Röhrle, "Spherical nilpotent orbits in positive characteristic”, Pacific J. Math. 237:2 (2008), 241-286. MR 2009f:14095 Zbl 05366370

[Grosshans 1992] F. D. Grosshans, "Contractions of the actions of reductive algebraic groups in arbitrary characteristic”, Invent. Math. 107:1 (1992), 127-133. MR 93b:14072 Zbl 0778.20018

[Humphreys 1995] J. E. Humphreys, Conjugacy classes in semisimple algebraic groups, Mathematical Surveys and Monographs 43, American Mathematical Society, Providence, RI, 1995. MR 97i:20057 Zbl 0834.20048 
[Knop 1995] F. Knop, “On the set of orbits for a Borel subgroup”, Comment. Math. Helv. 70:2 (1995), 285-309. MR 96c:14039 Zbl 0828.22016

[Panyushev 1994] D. I. Panyushev, "Complexity and nilpotent orbits", Manuscripta Math. 83:3-4 (1994), 223-237. MR 95e:14039 Zbl 0822.14024

[Panyushev 1999] D. I. Panyushev, "On spherical nilpotent orbits and beyond", Ann. Inst. Fourier (Grenoble) 49:5 (1999), 1453-1476. MR 2000i:14072 Zbl 0944.17013

[Panyushev and Röhrle 2005] D. Panyushev and G. Röhrle, "On spherical ideals of Borel subalgebras”, Arch. Math. (Basel) 84:3 (2005), 225-232. MR 2005k:14100 Zbl 1076.14061

[Premet 2003] A. Premet, "Nilpotent orbits in good characteristic and the Kempf-Rousseau theory", J. Algebra 260:1 (2003), 338-366. MR 2004i:17014 Zbl 1020.20031

[Springer 1985] T. A. Springer, "Some results on algebraic groups with involutions", pp. 525-543 in Algebraic groups and related topics (Kyoto/Nagoya, 1983), edited by R. Hotta, Adv. Stud. Pure Math. 6, North-Holland, Amsterdam, 1985. MR 86m:20050 Zbl 0628.20036

[Springer and Steinberg 1970] T. A. Springer and R. Steinberg, “Conjugacy classes”, pp. 167-266 in Seminar on Algebraic Groups and Related Finite Groups (Princeton, NJ, 1968/69), Lecture Notes in Mathematics 131, Springer, Berlin, 1970. MR 42 \#3091 Zbl 0249.20024

[Steinberg 1965] R. Steinberg, "Regular elements of semisimple algebraic groups", Inst. Hautes Études Sci. Publ. Math. 25 (1965), 49-80. MR 31 \#4788 Zbl 0136.30002

[Steinberg 1968] R. Steinberg, Lectures on Chevalley groups, Yale University, 1968. MR 57 \#6215 [Vinberg 1986] È. B. Vinberg, "Complexity of actions of reductive groups", Funk. Anal. i Prilozhen. 20:1 (1986), 1-13. In Russian; translated in Func. Anal. Appl. 20 (1986), 1-11. MR 87j:14077 Zbl 0601.14038

Received December 1, 2008. Revised May 7, 2009.

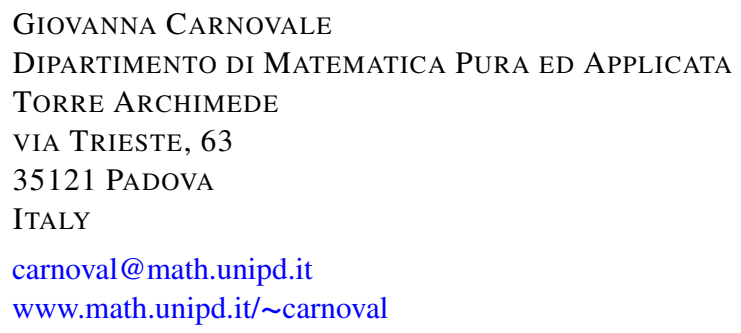

